\title{
Crewed Mission to Callisto Using Advanced Plasma Propulsion Systems
}

\author{
R.B. Adams ${ }^{1}$, G. Statham ${ }^{1,2}$, S. White1,2, B. Patton ${ }^{3}$, Y.C.F. Thio ${ }^{4}$, J. Santarius 5 , \\ R. Alexander ${ }^{1}$, S. Fincher ${ }^{1}$, T. Polsgrove ${ }^{1}$, J. Chapman ${ }^{1}$ and A. Philips ${ }^{1}$ \\ ${ }^{1}$ NASA Marshall Space Flight Center, Advanced Concepts Department, Huntsville, AL 35812, USA. \\ ${ }^{2}$ ERC Inc., 555 Sparkman Drive, Executive Plaza, Suite 1622, Huntsville, AL 35816, USA \\ ${ }^{3}$ NASA Marshall Space Flight Center, Propulsion Research Center, Huntsville, AL 35812, USA. \\ ${ }^{4}$ U.S. Department of Energy, Office of Fusion Energy Sciences, 19901 Germantown Road, Germantown, MD \\ 20874 \\ ${ }^{5}$ Institute of Fusion Technology, University of Wisconsin, Madison, WI 20874, USA
}

\begin{abstract}
This paper describes the engineering of several vehicles designed for a crewed mission to the Jovian satellite Callisto. Each subsystem is discussed in detail. Mission and trajectory analysis for each mission concept is described. Crew support components are also described. Vehicles were developed using both fission powered magneto plasma dynamic (MPD) thrusters and magnetized target fusion (MTF) propulsion systems. Conclusions were drawn regarding the usefulness of these propulsion systems for crewed exploration of the outer solar system.
\end{abstract}

\section{INTRODUCTION}

Administrator Daniel Goldin initiated the Revolutionary Aerospace Systems Concepts (RASC) activity starting in fiscal year 2001 to address conceptual design of systems and architectures for potential missions 25 to 40 years in the future. The results of these designs were to determine the technologies and infrastructure necessary to enable those missions. Consequently RASC studies were intended to take a "top-down" approach

In fiscal year 2002 RASC organized the Human Outer Planet Exploration (HOPE) group to investigate the possibility of crewed travel to the outer solar system. Three scenarios were considered, nuclear thermal propulsion using crewed and support vehicles, nuclear electric propulsion also using crewed and support vehicles and fusion propulsion using crewed vehicles only. The outer solar system from the asteroid belt and beyond was surveyed to select a suitable baseline destination point. Using assumptions on requirements and available technology three centers (GRC, LaRC and MSFC) designed vehicles to support a crewed trip to the outer solar system. The emphasis on this study was to gain a greater understanding of the challenges associated with these missions so that they can be addressed now.

\section{SELECTED DESTINATION}

A number of candidate destinations were considered before the final selection was made. The major selection criteria can be summarized as follows. The destination should be a body in the outer solar system, here defined as being beyond the orbit of Mars, it should be suitable for human surface exploration and it should contain some features of legitimate scientific interest. The requirement for human surface exploration ruled out any of the gas giant planets, Jupiter, Saturn, Uranus and Neptune, although not their extensive satellite systems. As a final criteria, it was desirable that the destination present a suitable level of challenge for the technology options considered.

Amongst the short-listed candidates were the following bodies. 
Ceres; the largest known asteroid, with a diameter of almost $1000 \mathrm{~km}$, located in the main asteroid belt. Scientific interest is largely focused on determining the composition and structure of the asteroid as a key to understanding the formation and development of the Solar System. Additional interest is prompted by asteroidal mining and planetary defense considerations. Due to its relatively close proximity and low mass, Ceres presents a simpler set of design challenges than more distant destinations.

Europa; the smallest of Jupiter's Galileian satellites and the second-closest to the planet itself. Scientific interest is largely prompted by the likely presence of a submerged ocean with tidal-heating, which could offer conditions conducive to the development of life. Europa's location, within the Jovian radiation belts, poses significant design challenges, particularly when contemplating human surface exploration.

Callisto; the second-largest Galilean satellite and the most distant from Jupiter itself. Scientific interest is prompted by the possibility of subsurface water. Callisto's distance from Jupiter places it in a significantly less hazardous radiation environment than Europa, potentially permitting human surface operations.

Titan; the largest satellite of Saturn and one of the largest in the Solar System. Scientific interest is considerable, in part because Titan appears to be more complex than other satellites, with a significant (and opaque) atmosphere, possibly offering conditions conducive to the development of life. The mission difficulty due to the relative remoteness of Saturn is offset by the absence of any significant radiation belts.

Chiron; an unusual minor planet, following an eccentric orbit with perihelion just outside the orbit of Saturn and aphelion just within that of Uranus. Scientific curiosity is prompted by Chiron's visible coma and variable brightness, which appear to indicate that volatile compounds are being vaporized from this, in other respects, asteroidal body. It is hypothesized that Chiron has only been resident in its present orbit for a relatively short period having previously been located in the (trans-Neptunian) Kuiper Belt. Access to Chiron could yield important data about the composition and structure of Kuiper Belt objects which are thought likely to be remnant objects from the Solar System's formation. Its remotenss and low temperature would pose significant technical problems.

Triton; the largest satellite of Neptune and the only significant satellite in the Solar System which follows a retrograde orbit. Scientific interest is due to its continuing seismic activity, as manifested by several "ice" volcanoes, and the likelyhood that Triton is a Kuiper Belt object, captured by Neptune in the relatively recent past. Triton's remotenss and low temperature pose significant design challenges.

The Jovian moon Callisto was selected because of the balance which it offers between scientific interest, design challenge severity and the level of hazard to human operations posed by the local environment.

\section{VEHICLE DESIGN}

Originally MSFC's task was to consider fusion concepts only for the crewed outer planet mission. The initial concepts selected were the Spherical Torus concept described by Williams et al., the Magnetized Target Fusion (MTF) concept by Thio et al. and the VISTA concept by Orth et al. These three concepts seemed to cover a broad range of fusion concepts applications from continuous magnetic confinement to inertial pulsed confinement. Fusion concepts employing antimatter were not considered as their level of development seemed too low to support a vehicle conceptual design.

One of the assumptions for this study was that the crew could only be subjected to one-year total of microgravity. Therefore any missions over a year would require an artificial gravity system. All of the above concepts were developed using a total mission time of one year and a crew stay time on the target planet of one 
month. The concepts above were capable of such a mission but some of the initial vehicle masses were untenable.

There was little design data on the Spherical Torus and VISTA concepts in the literature. The design team incorporated point designs found in the literature to complete preliminary vehicle designs to the selected destination. Unfortunately, these vehicles were non-competitive in part due to the fact that the engine parameters were optimized for different missions. Therefore MTF was the only reasonable option for further study.

Several different options for MTF were then considered. A Deuterium-Deuterium (D-D) reaction was considered to be the baseline with a Deuterium-Helium-3 shown as an advanced option. The fission concepts developed by the other centers required an 180-day crew time on planet. The stay time was defined by a desire to wait for Jupiter and Earth to come back into realignment and reduce total vehicle delta- $\mathrm{V}$ requirements. The study lead therefore asked for a fusion option with a 180 day stay time for purposes of consistency. While refining the mission design, it was determined that avoiding the artificial gravity requirement by forcing the mission duration to be under the one-year limit was more costly than accounting for the additional mass of an artificial gravity system. A total mission duration of 1 years was the final selection for the MTF missions. The one year mission required the vehicle to fly retrograde on both outbound and inbound legs. The 1 yr mission time eliminated this problem and reduced the acceleration requirements. The addition of an artificial gravity system was minor compared to the gains due to the decreased delta- $\mathrm{V}$ requirements of the longer mission.

From all of these considerations three MTF options were selected for further study; MTF D-D 30 day stay, MTF D-D 180 day stay; and MTF D-He3 180 day stay.

At the end of the study MSFC also investigated several technologies that hold promise to increase performance for the nuclear electric fission options. The baseline nuclear electric option was a solid core NERVA style reactor with a Brayton power conversion system and a magnetoplasmadynamic thruster assembly. MSFC investigated an option that replaced the reactor and power conversion system with a molten salt reactor and liquid metal rankine power conversion system. Additionally MSFC investigated a system that replaced the Brayton cycle with a magnetohydrodynamic power conversion system. These concepts require a five year mission time and split mission profile that the baseline case does not. These results are shown below.

\section{Payload Components}

The payload components of the HOPE vehicle consist of a Trans-Hab module, a surface habitat, a lander, and an ISRU plant. These components are responsible for providing a livable environment on the vehicle and on the surface of Callisto as well as collect scientific data. The Trans-Hab is the main living quarters for the sixcrew members. This module has a mass of approximately 40 metric tons with an additional 4 metric tons of consumables.

The surface habitat is an inflatable structure that can house three crewmembers on the surface of Callisto. It is responsible for providing shelter to the surface crew and will serve as a laboratory for surface experiments. A 250-kilowatt electric (1 megawatt thermal) reactor located 1 kilometer away will transmit power to the surface habitat for surface operations.

The lander is used as a crew and materials transit between the surface of Callisto and the orbiter. It will be capable of sending up to 40 metric tons down to the surface. It's fuel will come from resources existing on the surface of Callisto. 
Callisto is a desolate minor planet with more impact craters than any other object in the solar system. The crust of the moon dates back 4 billion years, that is around the time the solar system was formed. This ancient surface may be able to provide scientific information about our early solar system. In addition to scientific information Callisto may also be able to provide mission resources.

Callisto has a density of $1.86 \mathrm{gm} / \mathrm{cm}^{3}$, and is comprised of ice and rock. The $200 \mathrm{~km}$ thick crust is an icy layer that could be mined to produce water. Beneath the crust is believed to be an ocean of salt and water, $10 \mathrm{~km}$ deep. Because Callisto contains such an abundance of water, it can provide various resources. Through simple distillation and dissociation processes, oxygen and hydrogen can be produced for fuel and air. The In-Situ Resource Utilization (ISRU) unit will convert the icy reguleth of Callisto into water, LOX and liquid hydrogen at a rate equivalent to $21 \mathrm{~kg}$ of water per hour. This will provide enough propellant for the lander to rendezvous with the orbiter every thirty days. The ISRU will require a power of 215 kilowatts.

\section{Reaction Control System}

The reaction control system (RCS) is based on an oxygen and hydrogen chemical propulsion system. Twentyfour $500 \mathrm{lbf}$ thruster are located in two ring frames on the vehicles structure and provide full six-degrees-offreedom for attitude control, docking and spin control for artificial gravity. During the outbound and inbound portions of the fight, the manned vehicle is spun at approximately one-and-a-quarter revolutions a minute to simulate $25 \%$ of Earth gravity. To accomplish both of these tasks up to 85,000 pounds of liquid oxygen and liquid hydrogen propellant are stored separately from the main drive propellant. These propellants are maintained in a liquid state by the liberal application of multi-layer insulation with any additional heat leakage into the system removed with cryo-coolers powered by the main electrical system. To ensure that the propellants are at the proper gaseous temperatures and pressures required by these RCS thrusters, a series of run-tanks use electrical resistance heaters to convert the cryogenically stored liquid propellants into the gaseous propellants required for the RCS thrusters. These run tanks are sized to hold approximately one meter per second delta $\mathrm{V}$ of gaseous propellant, which is available on-demand to the RCS thruster. If additional propellant is required beyond the one meter per second stored in the run tanks, the run tanks become a conditioning plenum, converting liquid cryogenic liquid propellant into gaseous propellant during high demand attitude control and artificial gravity spin maneuvers. In addition to the main RCS thrusters are a series of smaller $50 \mathrm{lbf}$ hydrogen cold gas thrusters, which are used as needed for fine pointing and close proximity operations.

Figure 1. Baseline Vehicle RCS Schematic

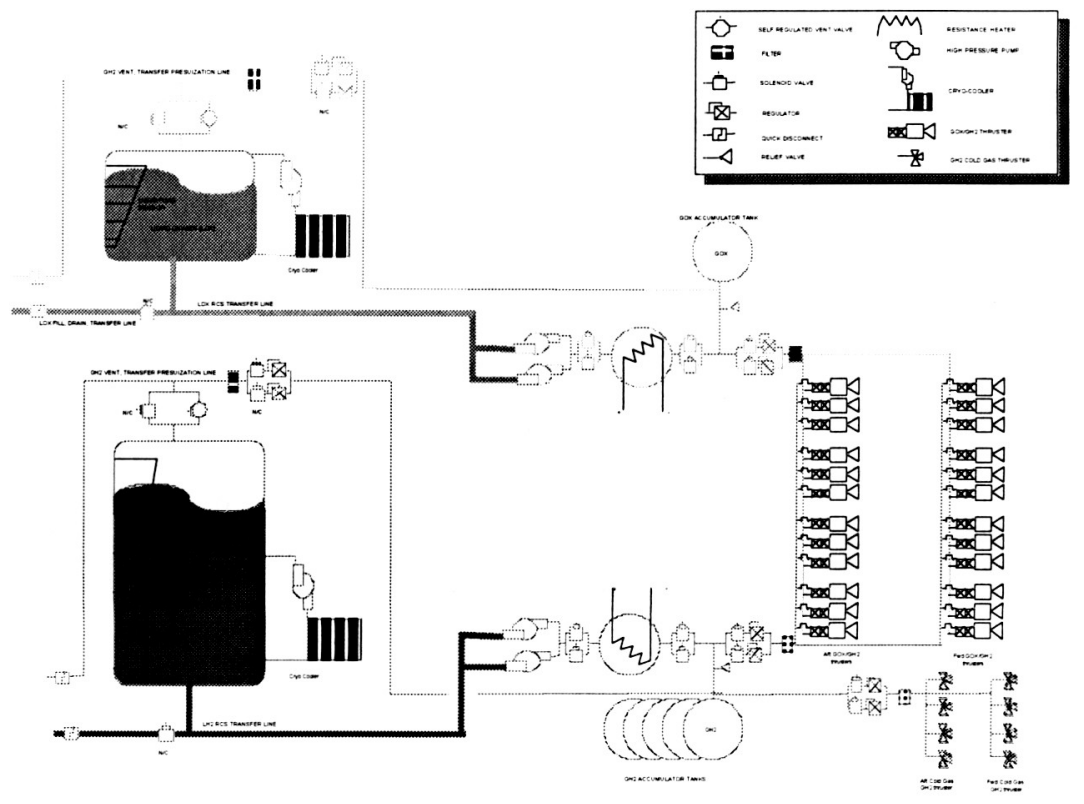




\section{Structural Components}

The main propellant tanks for this study were designed with the LVA (Launch Vehicle Analysis)[1] computer tool. The analysis was based on the assumption that the tank is launched fully loaded with hydrogen without a shroud and was not assumed to be pressure stabilized. LVA analyzed the tank using the full gamut of prelaunch, lift-off, and flight loads. The tanks have both fore and aft skirts that remain with the tank throughout the mission. The necessary docking equipment is launched within the skirts.

The main truss design was based on earlier manned Mars studies. With an on-orbit assembled truss, the designing loads are not the propulsion system thrust as is commonly thought. Most of the weight comes from the loads imparted during the ETO (Earth To Orbit) phase, assembly, and overall stiffness requirements. Therefore, truss sections can serve different space missions with little or no change.

The material used for both the main propellant tank and the truss was an unnamed study designated advanced material. This material was specified to have a strength three times greater than that of Aluminum.

\section{Thermal Control Systems}

There are three heat rejection systems for the HOPE vehicle: a low temperature radiator system for the avionics and crew, a medium temperature radiator for the power conversion system, and a high temperature radiator for the propulsion system waste heat. These radiators are necessary due to the need to maintain the systems within required temperature limits and in space the only method of dumping waste heat is through radiation heat transfer. All of the radiator systems use heat pipes to distribute the heat evenly across the panels. Figure xx is a simplified schematic of the thermal control system for the power conversion system and the MTF engine.

Figure 2. Schematic of Power Conversion and Engine Thermal Control Systems

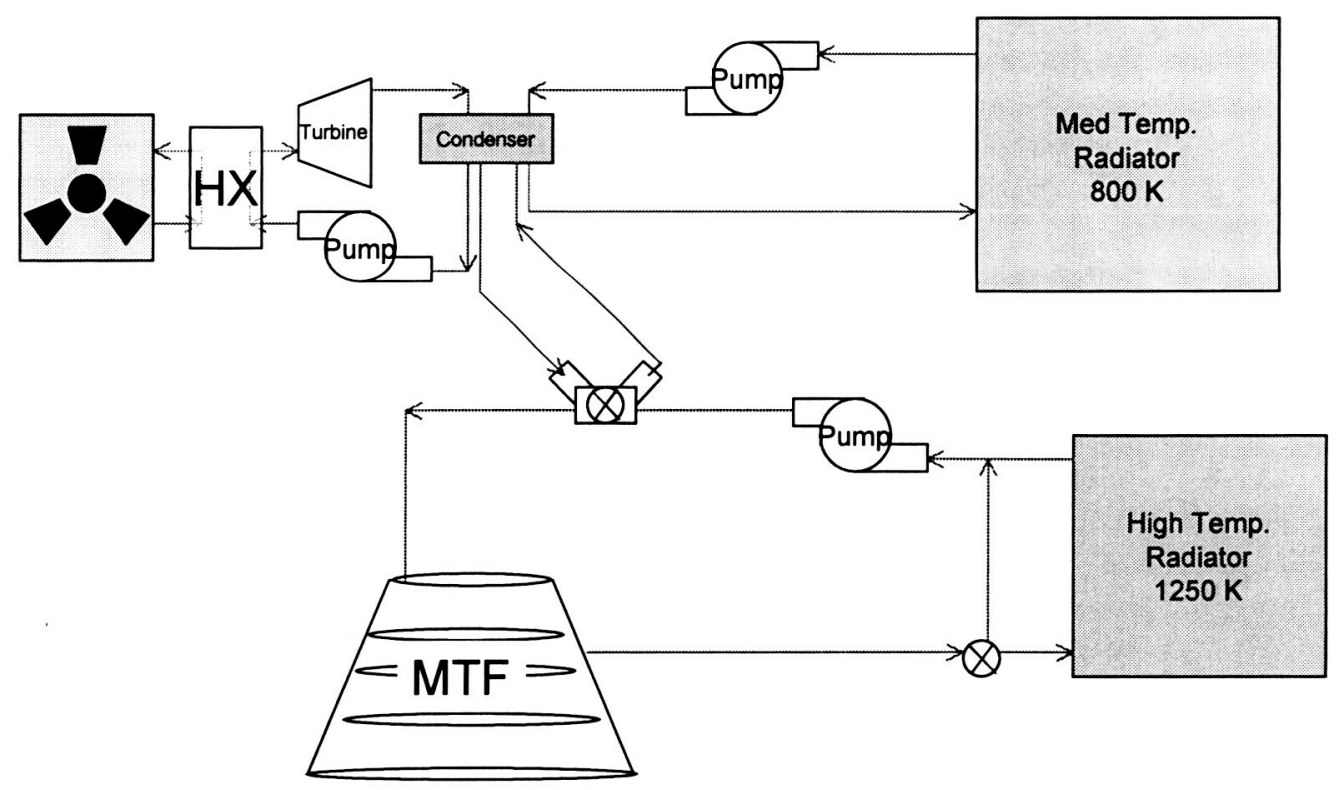


Thermal radiators for spacecraft systems need to be as lightweight as possible. However, some of the vehicle system requirements force the thermal radiator system to be heavier than would be necessary provided there was no other required functionality. The thermal radiator panels for advanced space vehicles of the type described in this document tend have large surface areas, thus requiring that they be deployed prior to usage. Deployment mechanisms add weight to the panel. Also reliability and safety requirements will likely add weight to the system. Many have attempted to analytically model these panels with various heat transport mechanisms and materials technology to determine the weight of the system. Near term technology incorporates composite panels with heat pipes imbedded in the panels.

Eventually, for the system analysts, the input to model the radiator simplifies to a unit area or "areal density". For advanced vehicles some analysts have estimated this unit weight be as low as $1 \mathrm{~kg} / \mathrm{m} 2$ and as high as 20 $\mathrm{kg} / \mathrm{m} 2$. Of course, technology assumptions for the study affect this number. The more advanced the technology assumption the lower the unit weight. For HOPE the design team assumed near-to-mid term technology which led to the assumption that the high temperature radiator panels, with the deployment mechanisms, safety, and reliability, have a unit mass of $10 \mathrm{~kg} / \mathrm{m}^{2}$ for single-sided or $5 \mathrm{~kg} / \mathrm{m}^{2}$ for two-sided panels, while the medium temperature panels were assumed to weigh $8 \mathrm{~kg} / \mathrm{m}^{2}\left(4 \mathrm{~kg} / \mathrm{m}^{2}\right)$. The low temperature radiator panels have an assumed unit mass of $3.7 \mathrm{~kg} / \mathrm{m}^{2}$ for two-sided panels. As a comparison, the International Space Station radiator panels weigh $8.5 \mathrm{~kg} / \mathrm{m}^{2}$ for two-sided panels.

Analyses performed to size the radiator panels assumed that the panels have a perfect view to space with no view of the Sun. Also the infrared emissivity (e) was assumed to be 0.9 . Fin effectiveness was ignored and the panel was assumed to be at a constant average temperature.

The medium temperature radiator panels reject heat from the power conversion system at $800 \mathrm{~K}$. The panels employ a combination of water and sodium-potassium heat pipes. The higher temperature $\mathrm{Na}-\mathrm{K}$ heat pipes are at the cooling fluid inlet to radiator with the water heat pipes near the cooler end. The heat rejection requirements vary with concepts analyzed, and panel surface areas and masses vary accordingly. These are summarized below in Table xx.

Table 1. Medium Temperature Radiator Parameters

\begin{tabular}{|l|l|l|l|}
\hline \multicolumn{5}{|c|}{ Medium Temperature } & Radiator \\
\hline Concept & D-D MTF 30 Day Stay & D-D MTF 180 Day Stay & D-He3 180 Day Stay \\
\hline Heat Rejection Requirement (MW) & 24.1 & 45.8 & 33.9 \\
\hline Heat Rejection Temperature (K) & 800 & 800 & 800 \\
\hline Radiator Total Surface Area (m²) & 1156.6 & 2192.3 & 1622.7 \\
\hline Radiator Mass (kg) & 4614.5 & 10961.5 & 6490.9 \\
\hline
\end{tabular}

The high temperature radiator panels reject the heat from the propulsion system. These panels use heat pipes that have Lithium as a working fluid. The temperature of these panels is assumed to be $1250 \mathrm{~K}$. The heat is collected in the propulsion system using a high temperature molten salt (FLiBe). The FLiBe passes through a heat exchanger to which the evaporator ends of the heat pipes are connected where it is cooled. The cooled 
fluid flows back in the engine completing the thermal control circuit. As with the medium temperature radiator the heat rejection requirements vary with the vehicle concepts and are summarized below in Table xx.

Table 2. High Temperature Radiator parameters

\begin{tabular}{|l|l|l|l|}
\hline \multicolumn{5}{|l|}{ High Temperature } & Radiator \\
\hline Concept & D-D MTF 30 Day Stay & D-D MTF 180 Day Stay & D-He3 180 Day Stay \\
\hline Heat Rejection Requirement (MW) & 261.9 & 497.9 & 176.5 \\
\hline Heat Rejection Temperature (K) & 1250 & 1250 & 1250 \\
\hline Radiator Total Surface Area (m²) & 2103.3 & 3998.6 & 1417.5 \\
\hline Radiator Mass $(\mathrm{kg})$ & 10516.5 & 19993 & 7087.3 \\
\hline
\end{tabular}

Cryogenic refrigeration systems are used to maintain the LH2 propellants during the transit to Calisto. Cryogenic hydrogen has a boiling point of about $20 \mathrm{~K}$ so any amount of heat leak into the tank affects the mission through propellant boil-off. For long term missions passive insulation systems are massive accounting for the propellant boil-off during the mission. Zero boil-off (ZBO) systems use a combination of active and passive thermal control to provide a minimum mass solution. ZBO systems comprise subsystems that include the refrigerator, power system, controller, thermal radiator to reject the waste heat from the system and the insulation system, which is composed of multi-layer insulation and foam. The combination of the insulation and refrigeration system is optimized to attain the minimum system mass taking into account the propellant.

The ZBO system sized for the HOPE mission uses today's cryo-cooler technology assuming 2-stage coolers for the $\mathrm{LH}_{2}$ tanks. The mass and power calculations were based on research and analyses performed by NASA's Glenn Research Center, Marshall Space Flight Center and Ames Research Center. The analysis methodology takes into account the mass of the propellant, the size (surface area) of the propellant tanks, the number of propellant tanks, the type of propellant, tank pressure, and environmental conditions. Using this methodology a ZBO system was adequately sized to maintain the required cryogenic propellant for the HOPE mission.

\section{Auxiliary Power}

For the HOPE/RASC studies, a liquid metal potassium Rankine system was selected for the power conversion module; for both the solid pin lithium cooled reactor (LMR) reactor and the Molten Salt reactor concepts.

Potassium Rankine systems are actually quite well characterized, as all of the major components (turbine, condenser, alternator and heat exchangers) have undergone many hours of extensive ground testing. One advantage of Rankine systems is that the heat is typically rejected at much higher temperatures (approximately $900{ }^{\circ} \mathrm{K}$ versus $425{ }^{\circ} \mathrm{K}$ for a Brayton cycle). Thus, the radiator mass tends to be much smaller for Rankine systems. This approximate "doubling" of the heat rejection temperature allows the radiator mass to be reduced approximately by a factor of 16 , since $2^{4}=16$.

$$
Q=\sigma A\left(T_{r e j}^{4}-T_{o}^{4}\right) \quad \text { Eq. } 1
$$


A picture of a liquid metal Rankine conversion system is shown below in Figure 3.

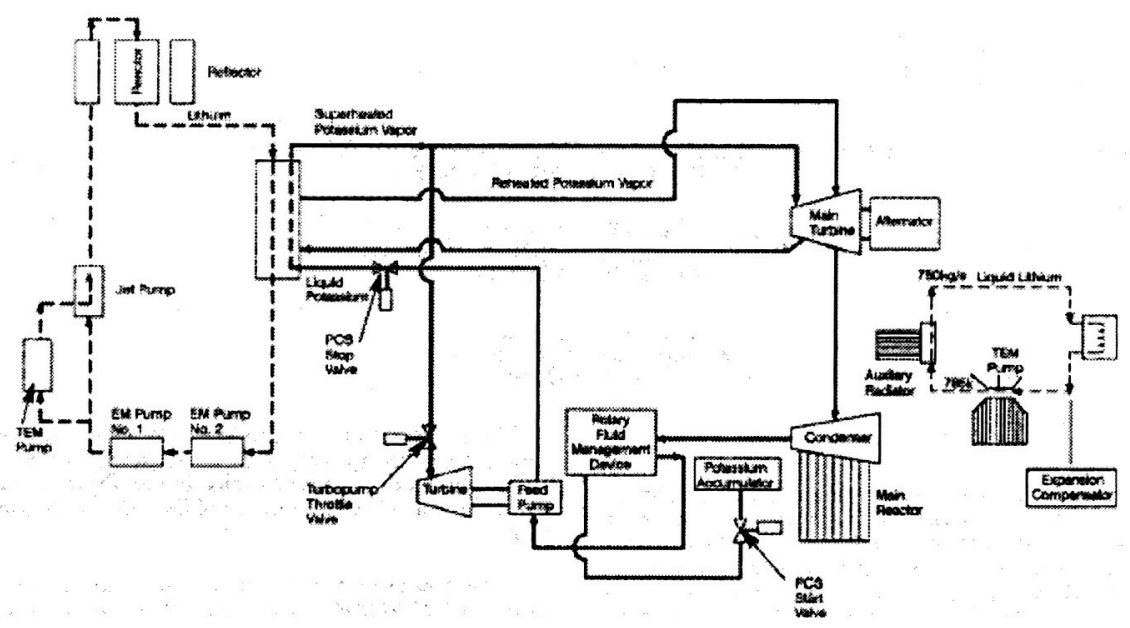

Figure 3. Liquid metal Rankine conversion system

A typical space system layout is shown in Figure 4.

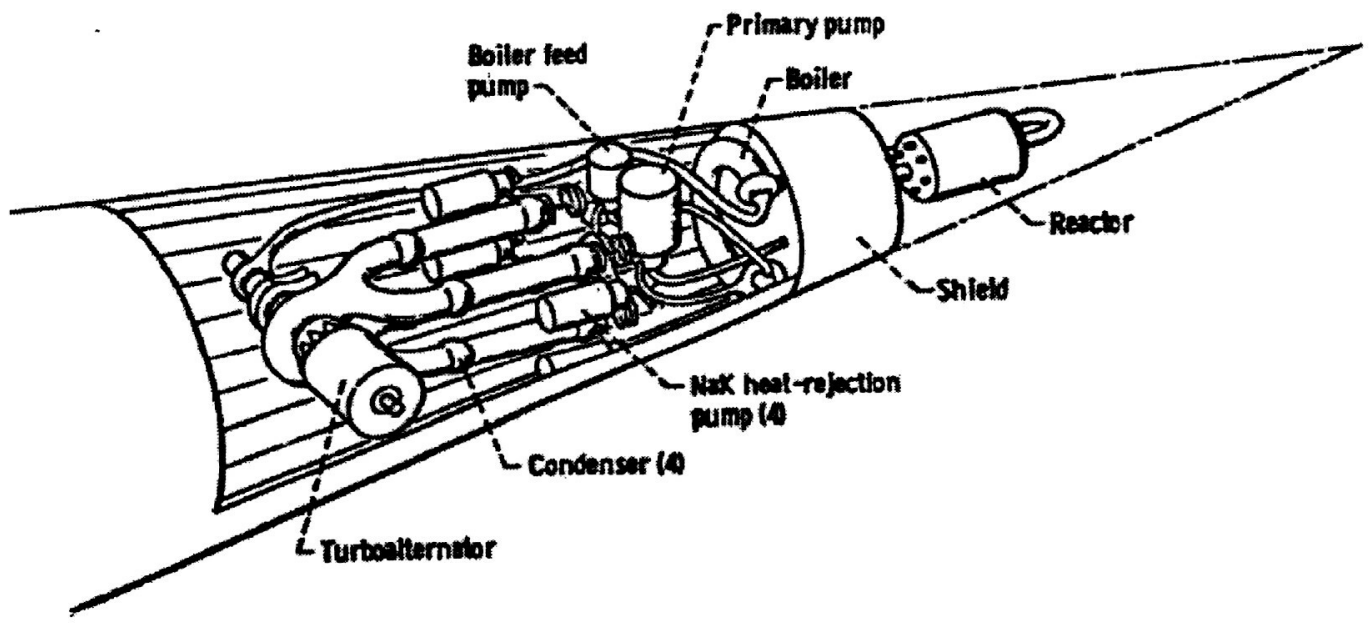

Figure 4. Liquid metal Rankine system NEP vehicle layout

The liquid metal potassium Rankine cycle is similar to the typical steam cycle employed by the commercial utility industry, except that potassium is used as the working fluid. 
The computer program ALKSYS was used for the APU design. The following discussion is taken directly from the ALKSYS users manual (ORNL-TM-10427).

"In the power conversion system, the principal flow of dry, saturated potassium vapor leaving the boiler of the lithium-cooled reactor is diverted to the turbine of the turbo feed pump. As the vapor expands through the main turbine, inter-stage and external separators are used to maintain the liquid content of the stream at $<12 \%$ to avoid the potential for erosion of the turbine blades. Upon exhausting from the turbine, the vapor is condensed in tapered annular spaces surrounding the evaporator sections of the radiator heat pipes. Condensate is drawn from the small ends of the condensing annuli by a jet pump that is driven by a small stream of liquid taken from the discharge of the turbine driven feed pump. Liquid discharging from the jet pump flows to the intake of the feed pump and is then pumped through the shell sides of a specified number of feed heaters $(0-3)$ back to the boiler. One of the heaters is heated by feed pump turbine exhaust; other heaters, if more than one are specified, are heated by vapor extracted form appropriate stages in the main turbine or taken from the boiler outlet line if necessary, to provide the specified boiler feed temperature.

Turbine blade tip velocity is a parameter that is dependent upon the strength of the turbine rotor material and is treated as an input variable in the model to allow evaluation of the effects of advanced rotor materials. This parameter has a strong effect on turbine size and rotational speed, and therefore, on the mass of the turbine and the generator.

Most of the required input information for the ALKSYS code operation pertains to the power conversion sub-model. Input includes turbine inlet and outlet temperatures, dry stage efficiency for the turbine, and the number of stages of feed heating.

The code outputs a complete mass and energy balance for the power conversion subsystem, as well as mass estimates for the major components.

The major heat rejection load from the power system is from the power turbine condenser. The heat is rejected by a heat pipe radiator operating at a temperature somewhat lower than that of the condenser. A smaller heat rejection load from cooling of the reactor shield and the turbine generator, is rejected by a low temperature heat pipe radiator.

The geometry of the radiators depends upon the power system rating. Input requirements for the heat rejection sub-model include launch vehicle bay dimensions and the operating temperature of the low temperature radiator. Thermal loads for both parts of the radiator are provided by the power conversion sub-model, which provides dimensional information land the estimated mass of the heat rejection system.

The solid core reactor design is based on a fast spectrum, metallic-clad rod fuel element containing UN pellets. The primary coolant is lithium, hence it is similar to the SP-100 genre. The ALKYS code limits the peak pellet burn-up to 10 at $\%$ and the peak heat flux to $80 \mathrm{~W} / \mathrm{cm}^{2}$. Rod diameter is determined by heat flux and burnup, but it is limited to $0.64 \mathrm{~cm}$ for mechanical stability. Reactor control is provided by in-core assemblies and by rotatable drums located around the core periphery. ASTAR-811C is used for the fuel rod cladding and structural components operating at temperatures above $1100 \mathrm{~K}$, and $\mathrm{Nb}-$ $1 \% \mathrm{Zr}$ is used at lower temperatures. The design stress is two thirds of that which produces $1 \%$ creep during the system lifetime. 
Two options are available in the ALKSYS code for estimating shield size and mass. In the two-pi option, the shield configuration and dimensions are selected to provide a fast neutron fluence of $10^{13}$ neutrons $/ \mathrm{cm}^{2}$ and a total gamma dose of $10^{6} \mathrm{rad}$ at a plane that is $15 \mathrm{~m}$ from and perpendicular to the axis of the cylindrical reactor vessel. The shield is thickest along the axis that faces the payload, but it also covers the sides of the reactor to reduce scattered radiation. The two-pi shield utilizes layers of lithium hydride in a honeycomb matrix and tungsten. The second option utilizes an alternative shield configuration consisting of a thin layer of lithium hydride on all exposed surfaces of the reactor and a shadow shield of lithium hydride and tungsten that faces the payload. The shadow shield is selected to provide a prescribed fluence of fast neutrons and gamma dose at a payload dose plane with given diameter and distance from the reactor."

The ALKSYS code was used to estimate masses of the various subsystems of the vehicle. The lithium cooled UN reactor option was selected to provide mass estimates of the reactor and shield. The shadow shield configuration was selected with the same radiation requirements as imposed for the two-pi option as discussed above.

\section{Trajectory Simulation}

The computer program VARITOP [1,2] was the primary tool used for trajectory analysis in the HOPE study. VARITOP is a low-thrust trajectory optimization program that was developed by the Jet Propulsion Laboratory (JPL) and first used in the 1970's. It is now widely used at JPL, Glenn Research Center (GRC), and Marshall Space Flight Center for preliminary mission design studies. It's sister program, SEPTOP, was used to provide trajectory support for the Deep Space 1 mission.

For the heliocentric phase of the trajectory, VARITOP is a two-body, Sun-centered analysis tool. The planets are assumed massless, and targeting constraints match planetary positions and velocities relative to the Sun. The problem solution involves numerical integration of the state and costate or variational equations and the solution of a two-point boundary value problem to satisfy terminal constraints. The optimization, based on the Calculus of Variations, allows users to optimize many design variables. Departure date, flight time, and power required were some of the variables optimized in the HOPE mission analysis.

VARITOP also offers several endpoint bias conditions that address the planetary departure and arrival phases of the trajectory. Of these, the most useful for this study is the low-thrust escape or capture spiral bias. For this option, it is assumed that the spacecraft departs from or is captured into a circular orbit around the planet using only the low thrust propulsion system. The formulation of the performance equations for these spiraling escape or capture maneuvers can be found in the paper, "Performance Computations with Pieced Solutions of Planetocentric and Heliocentric Trajectories for Low-Thrust Missions," by Melbourne and Sauer [3].

The current version of VARITOP, VARITOP 2000, will do one spiral capture maneuver at the target body. The HOPE mission requires two. The capture maneuver at Jupiter consists of a spiral descent to the mean altitude of Callisto's orbit around Jupiter, and then another spiral around Callisto to descend to an orbit that is $500 \mathrm{~km}$ above Callisto's surface. For piloted vehicles, this same problem is encountered upon Callisto orbit departure, where two departure spiral maneuvers are required to escape Jupiter. The additional propellant needed for the Callisto spirals is calculated outside of VARITOP for the MPD missions. The fusion-powered missions required all spirals to be calculated outside of VARITOP. 
For some combinations of vehicle acceleration and spiral initial or final altitude, the VARITOP spiraling approximations are not valid. This was the case for the fusion-powered missions considered. For those three fast missions, the initial vehicle acceleration was between 0.0005-0.0008 g's, with higher accelerations at subsequent mission phases due to propellant depletion. Figure 5 [4] was used to approximate the velocity increment needed to escape from or capture into planetocentric orbits for cases where VARITOP could not. This figure was first generated by Sandorff [5] and is based on the work of Irving [6] and Tsien [7]

Figure 5. Penalty for low thrust in escape maneuver from satellite orbit, as compared to impulsive thrust (tangentially directed thrust) [4]

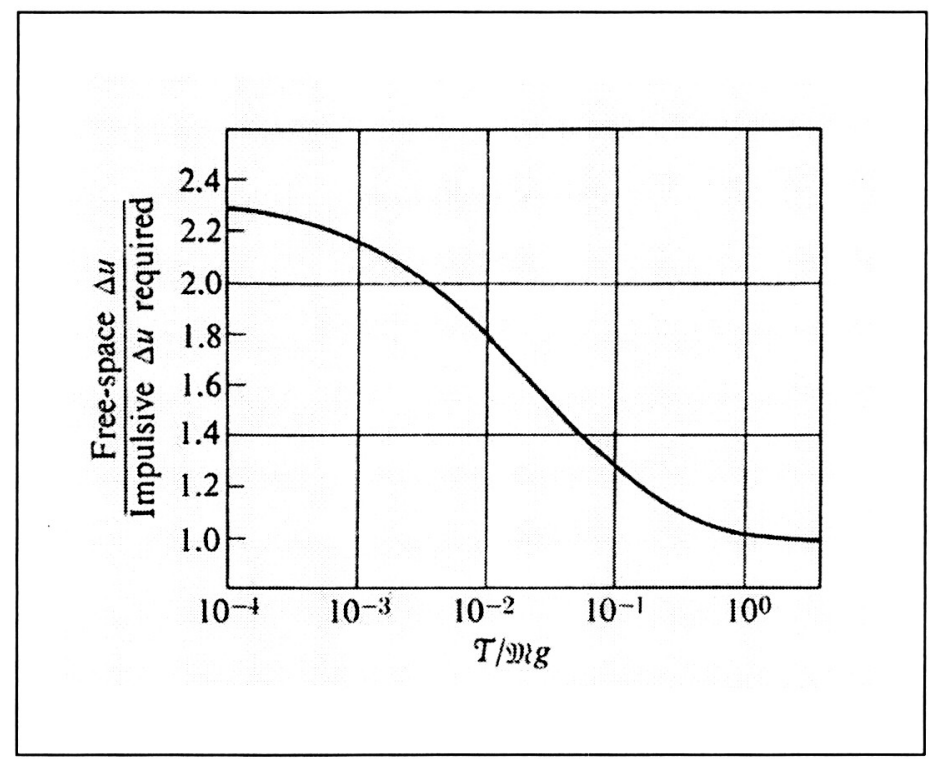

\section{Trajectory Summaries}

\section{Fusion Missions}

Each trajectory is optimized to give maximum payload for a given set of constraints and independent variables. For the fusion-powered missions, the mission time was constrained to 650 days (excluding Earth escape and capture times). This time was selected though a series of compromises. The team wanted to do a short mission to demonstrate the capabilities of the MTF device, however, maximizing payload mass typically means extending the trip time. Another consideration was the spacecraft's distance from the sun. Missions at 650 days or slightly less generally maintained a distance of $1 \mathrm{AU}$ or more from the sun throughout the mission, while slightly longer missions got closer, meaning more radiation for the crew. In an attempt to limit the harmful radiation dose from the Sun, maintain a relatively short mission duration, and maximize payload delivery, a 650-day mission was selected.

While the mission duration was fixed, the flight time for the outbound and return trajectory legs was not. The final optimized missions have return trajectory legs that are shorter than the outbound trajectories. This is an expected result because the vehicle has a much higher thrust-to-weight ratio on the return leg since most of the propellant has been used and most of the payload is left at Jupiter. 
VARITOP is also allowed to choose the power level and departure date that will optimize the mission performance. The final results for the three fusion-powered vehicles considered are given below.

\section{D-D MTF 30-day Stay}

Table 3 D-D MTF 30-day Stay Summary Information

\begin{tabular}{|llll|}
\hline Total Mission Duration $\sim 654$ days & Mission Timeline & & \\
Outbound Leg Departs 4/22/2045 & & Time & Mass \\
Flight to Callisto $\sim 331$ days & & (days) & $(\mathrm{mT})$ \\
Time in Callisto Orbit $\sim 33$ days & Depart L1 Station & 0 & 650 \\
Total time thrusting $\sim 258$ days & Thrust off & 51 & 630 \\
Returns without Surface Habitat, ISRU, and Transport & Thrust on & 240 & 630 \\
$(120 \mathrm{mt}$ total $)$ & Arrive Callisto Orbit & 331 & 595 \\
Isp $=70,400$ sec & Depart Callisto Orbit & 365 & 475 \\
Jet Power $=1.072 \mathrm{GW}$ & Thrust off & 440 & 445 \\
Propulsion System Specific Mass $=0.046 \mathrm{~kg} / \mathrm{kW}$ & Thrust on & 614 & 445 \\
Initial Acceleration $=0.0005 \mathrm{~g}$ 's & Arrive L1 Station & 654 & 430 \\
Final Acceleration $=0.0007 \mathrm{~g}$ 's & & & \\
\hline
\end{tabular}


Figure 6 Trajectory Graph for D-D MTF 30-day Stay Option

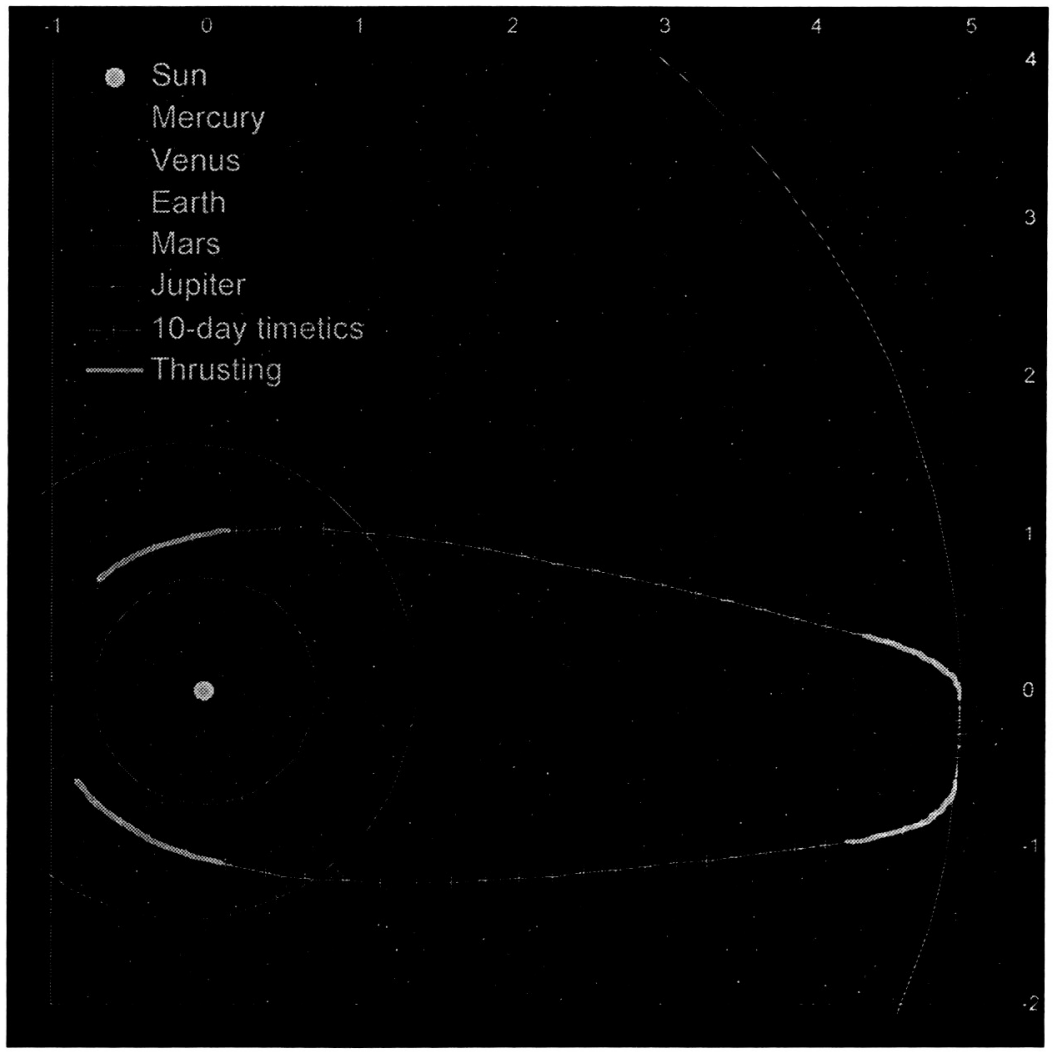

D-D MTF 180-day Stay

Table 4. D-D MTF 180-day Stay Summary Information

\begin{tabular}{|llll|}
\hline Total Mission Duration $\sim 652$ days & Mission Timeline & & \\
Outbound Leg Departs 4/26/2045 & & Time & Mass \\
Flight to Callisto $\sim 249$ days & & (days) & $(\mathrm{mT})$ \\
Time in Callisto Orbit $\sim 183$ days & Depart L1 Station & 0 & 750 \\
Total time thrusting $\sim 212$ days & Thrust off & 45 & 717 \\
Returns without Surface Habitat, ISRU, and Transport & Thrust on & 177 & 717 \\
$(120$ mt total) & Arrive Callisto Orbit & 249 & 664 \\
Isp $=70,400$ sec & Depart Callisto Orbit & 432 & 544 \\
Jet Power $=2.038$ GW & Thrust off & 492 & 499 \\
Propulsion System Specific Mass $=0.017 \mathrm{~kg} / \mathrm{kW}$ & Thrust on & 617 & 499 \\
Initial Acceleration $=0.0008 \mathrm{~g}$ 's & Arrive L1 Station & 652 & 473 \\
Final Acceleration $=0.0013 \mathrm{~g}$ 's & & & \\
\hline
\end{tabular}


Figure 7. Trajectory Graph for D-D MTF 180-day Stay Option

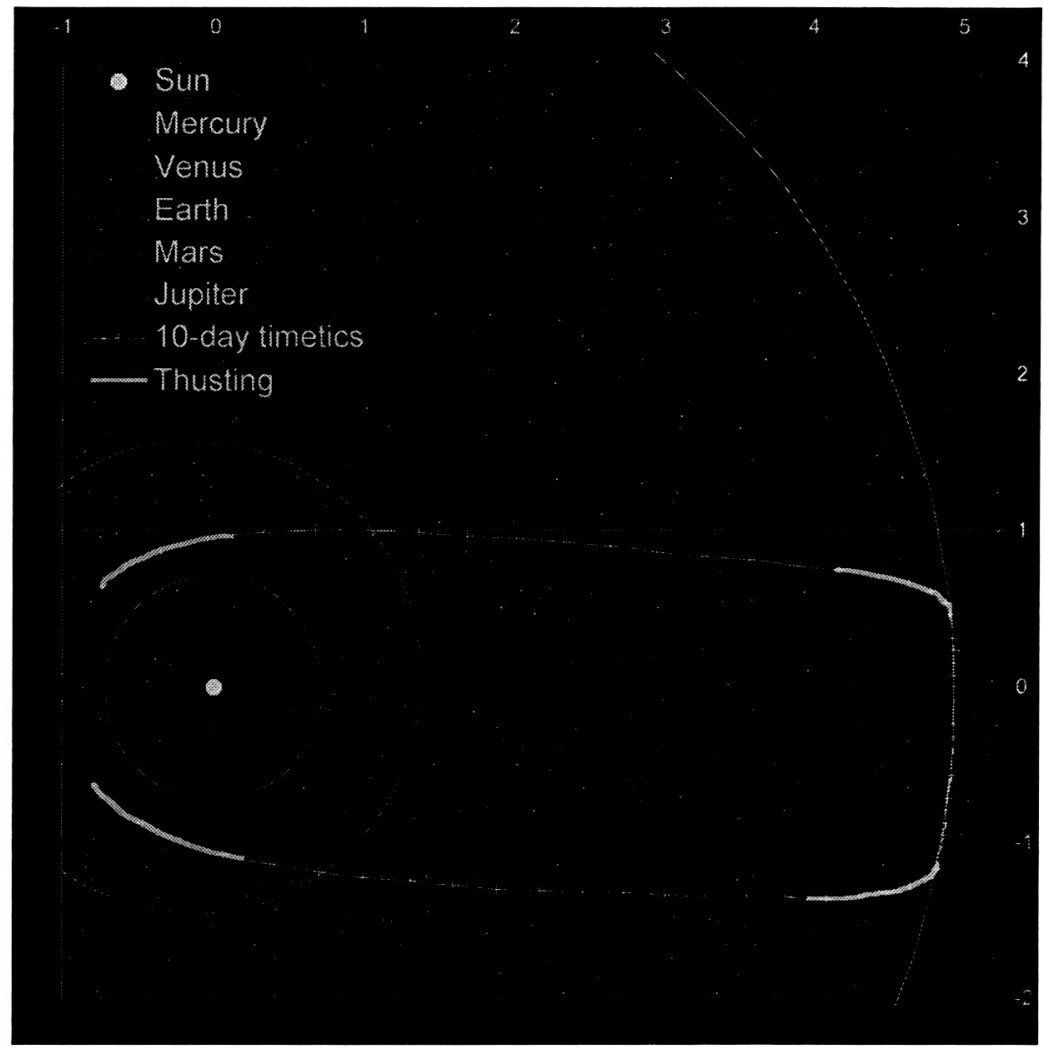

D-He ${ }_{3}$ MTF 180-day Stay

Table 5. D-He ${ }_{3}$ MTF 180-day Stay Summary Information

\begin{tabular}{|llll}
\hline Total Mission Duration $\sim 652$ days & Mission Timeline & & \\
Outbound Leg Departs 4/27/2045 & & Time & Mass \\
Flight to Callisto $~ 249$ days & & (days) & $(\mathrm{mT})$ \\
Time in Callisto Orbit $\sim 183$ days & Depart L1 Station & 0 & 700 \\
Total time thrusting $\sim 215$ days & Thrust off & 46 & 671 \\
Returns without Surface Habitat, ISRU, and Transport & Thrust on & 176 & 671 \\
$(120$ mt total) & Arrive Callisto Orbit & 249 & 626 \\
Isp $=77,000$ sec & Depart Callisto Orbit & 432 & 506 \\
Jet Power $=2.071 \mathrm{GW}$ & Thrust off & 493 & 468 \\
Propulsion System Specific Mass $=0.018 \mathrm{~kg} / \mathrm{kW}$ & Thrust on & 616 & 468 \\
Initial Acceleration $=0.0008$ g's & Arrive L1 Station & 652 & 445 \\
Final Acceleration $=0.0013 \mathrm{~g}$ 's & & &
\end{tabular}


Figure 8 Trajectory Graph for D-He3 MTF 180-day Stay Option

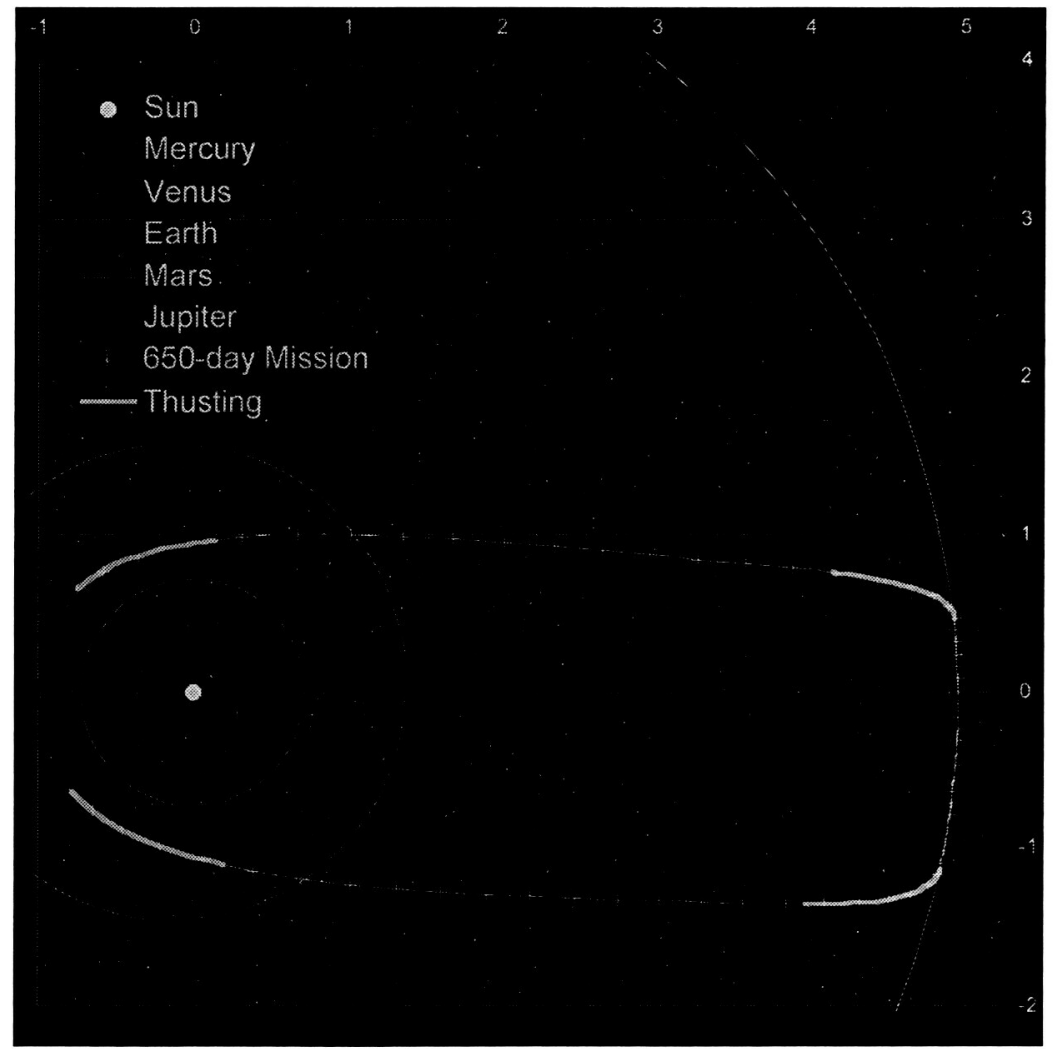

\section{MPD Missions}

There are two vehicles for each of the MPD missions. Cargo (surface habitat, ISRU, and transport vehicle) is predeployed on a separate vehicle from the crew. The trajectory optimization method is the same for both vehicles. The flight time for both vehicles was modeled after the Glenn Research Center team's MPD missions. This was done so that the effects of different technologies between the cases could be analyzed. For these trajectories, VARITOP was able to approximate the Jupiter capture and escape spirals, while the Earth and Callisto capture and escape spirals had to be approximated outside of the program. The Earth spirals, because the altitude at L1 in combination with the thrust-to-weight ratio of the vehicle was outside of the applicability of VARITOP's approximation technique, and Callisto, because VARITOP can only spiral about the destination planet of Jupiter.

\section{MPD Thrusters}

The Magnetoplasmadynamic (MPD) thruster is a member of the electromagnetic class of electric propulsion systems. These thrusters use electromagnetic forces to accelerate its plasma propellant to speeds in excess of 50 $\mathrm{km} / \mathrm{sec}$. With specific impulse values reaching 5,000 seconds and thrust values up to 2 Newton, the MPD thruster has a very competitive performance when compared to other electric propulsion systems. 
An MPD thruster makes use of coaxially arranged electrodes consisting of a center spike cathode and an outer annular anode. An inert gas such as Xenon is flown between the electrodes via a rear injector plate. An electric potential is applied across the electrodes great enough to ionize the gas. Once ionized, the gas becomes electrically conductive and a current flows from the anode through the gas and to the cathode. This current, along with the electrode geometry, produces an induced circular magnetic field between the electrodes. The resulting Lorentz force caused by the electric and magnetic field accelerates the ionized gas (plasma) in a direction perpendicular to both the electric and magnetic fields. It is this force that is responsible for producing thrust.

The arc discharge between the two electrodes produces a large amount of heat that raises the temperature of the thruster. Most of this heat must be removed by a cooling system to protect the materials. Some MPD thrusters are designed to use this heat to produce additional thrust by thermodynamic expansion. Some MPD thrusters are also augmented by including an external magnetic coil wrapped around the thruster. This coil increases the field strength of the induced circular magnetic field in between the electrodes and can improve performance.

Figure 9. Schematic of MPD thruster

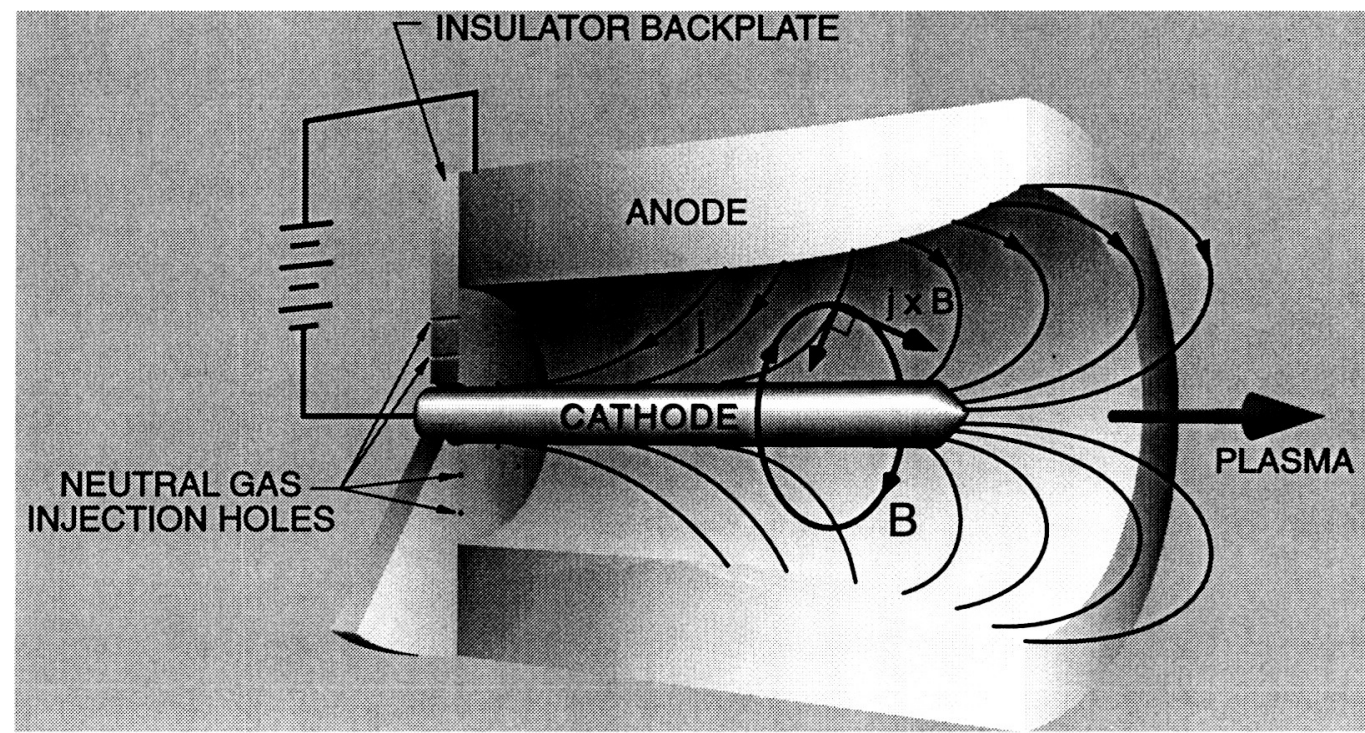

Advances in MPD performance are being pursued by going to higher power systems and using Hydrogen gas as the propellant. Because hydrogen has a light molecular weight, it can exhaust at a higher velocity and result in larger values of specific impulse. It is speculated that specific impulse values as high as 10,000 seconds may be achievable, but this remains to be seen. Although most MPD thrusters tested to date operate at hundreds of Kilowatts, it may be possible to push their power levels as high as Megawatts. The efficiency of these thrusters ranges between $30 \%$ to $50 \%$ of the input power being converted into jet power.

The limiting factors of the MPD thruster are based on the plasma physics inside the electrical discharge. For an MPD thruster, most of the losses are associated with the thermal energy created by the high current passing through the plasma. The plasma has an electrical resistance based on the level of ionization that is determined by the voltage across the electrodes. The higher the voltage, the more current passes through the plasma, the stronger the induced magnetic field, and the more thrust produced. Performance is limited by the geometry of the electrodes, the electrical properties of the propellant, and the magnetic Reynolds number.

In order for the propellant to be ionized, a strong enough electric field must be applied. This is essentially a function of the density of the gas, the voltage potential across the electrodes, and the distance between the electrodes. These relations govern the mass flow rate, power, and size combinations available to a thruster. 
The Specific Impulse of the thruster is limited by the magnetic Reynolds number. This is a nondimensional number that represents the ratio of the convective properties of a magnetic field in moving plasma with the ability of a magnetic field to diffuse through the plasma. For plasma moving through a stationary magnetic field, a magnetic Reynolds number less than one indicates that the plasma can move freely through the magnetic field. The limit of this case is when the magnetic Reynolds number equals unity. In this case, the speed of the moving plasma and the rate at which the magnetic field can diffuse through the plasma are equal. If the magnetic Reynolds number becomes greater than unity, the magnetic field is dragged along with the moving plasma and produces a drag force on the plasma flow. This will result in a loss of performance. This may also lead to instabilities within the plasma that can further reduce performance and thruster life.

The life limiting parts of the thruster are the electrodes. The high currents cause sputtering of the electrode material that eventually reduces performance to an unacceptable level. The best erosion rate achievable with current models is 0.5 nanograms per ampere per second or a sputtering of one atom for every $5 \mathrm{E}^{6}$ ions collected on the electrodes. This limits the integrated impulse to approximately $10^{6}$ Newton seconds.

The final results for the two MPD vehicles considered are given below.

MSR-LMR-MPD

Table 6 Cargo MSR-LMR-MPD 120-day Stay Summary Information

\begin{tabular}{|llll|}
\hline Total Mission Duration $\sim 1120$ days & Mission Timeline & & \\
Outbound Leg Departs 9/2/2041 & & Time & Mass \\
Total time thrusting $\sim 588$ days & & (days) & $(\mathrm{mT})$ \\
Isp $=8,000 \mathrm{sec}$ & Depart L1 Station & 0 & 456 \\
Total Power $=11 \mathrm{MW}$ & Thrust off & 329 & 390 \\
Jet Power $=7 \mathrm{MW}$ & Thrust on & 861 & 390 \\
Propulsion System Specific Mass $=5.36 \mathrm{~kg} / \mathrm{kW}$ & Arrive Callisto Orbit & 1120 & 337 \\
\hline
\end{tabular}


Table 7 MSR-LMR-MPD 120-day Stay Summary Information

\begin{tabular}{|llll|}
\hline Total Mission Duration $\sim 1661$ days & Mission Timeline & & \\
Outbound Leg Departs 11/19/2044 & & Time & Mass \\
Flight to Callisto $\sim 832$ days & & $($ days $)$ & $(\mathrm{mT})$ \\
Time in Callisto Orbit $\sim 120$ days & Depart L1 Station & 0 & 1072 \\
Total time thrusting $\sim 812$ days & Thrust off & 34 & 1049 \\
Returns with $13 \mathrm{mt}$ less consumables & Thrust on & 60 & 1049 \\
Isp $=8,000$ sec & Thrust off & 284 & 897 \\
Total Power $=37 \mathrm{MW}$ & Thrust on & 593 & 897 \\
Jet Power $=24 \mathrm{MW}$ & Arrive Callisto Orbit & 832 & 735 \\
Propulsion System Specific Mass $=5.36 \mathrm{~kg} / \mathrm{kW}$ & Depart Callisto Orbit & 952 & 722 \\
Initial Acceleration $=0.0001$ g's & Thrust off & 1145 & 591 \\
Final Acceleration $=0.0001 \mathrm{~g}$ 's & Thrust on & 1540 & 591 \\
& Arrive L1 Station & 1661 & 509 \\
\hline
\end{tabular}


Figure 10 Trajectory Graph for SCR-LMR-MPD 120-day Stay Option

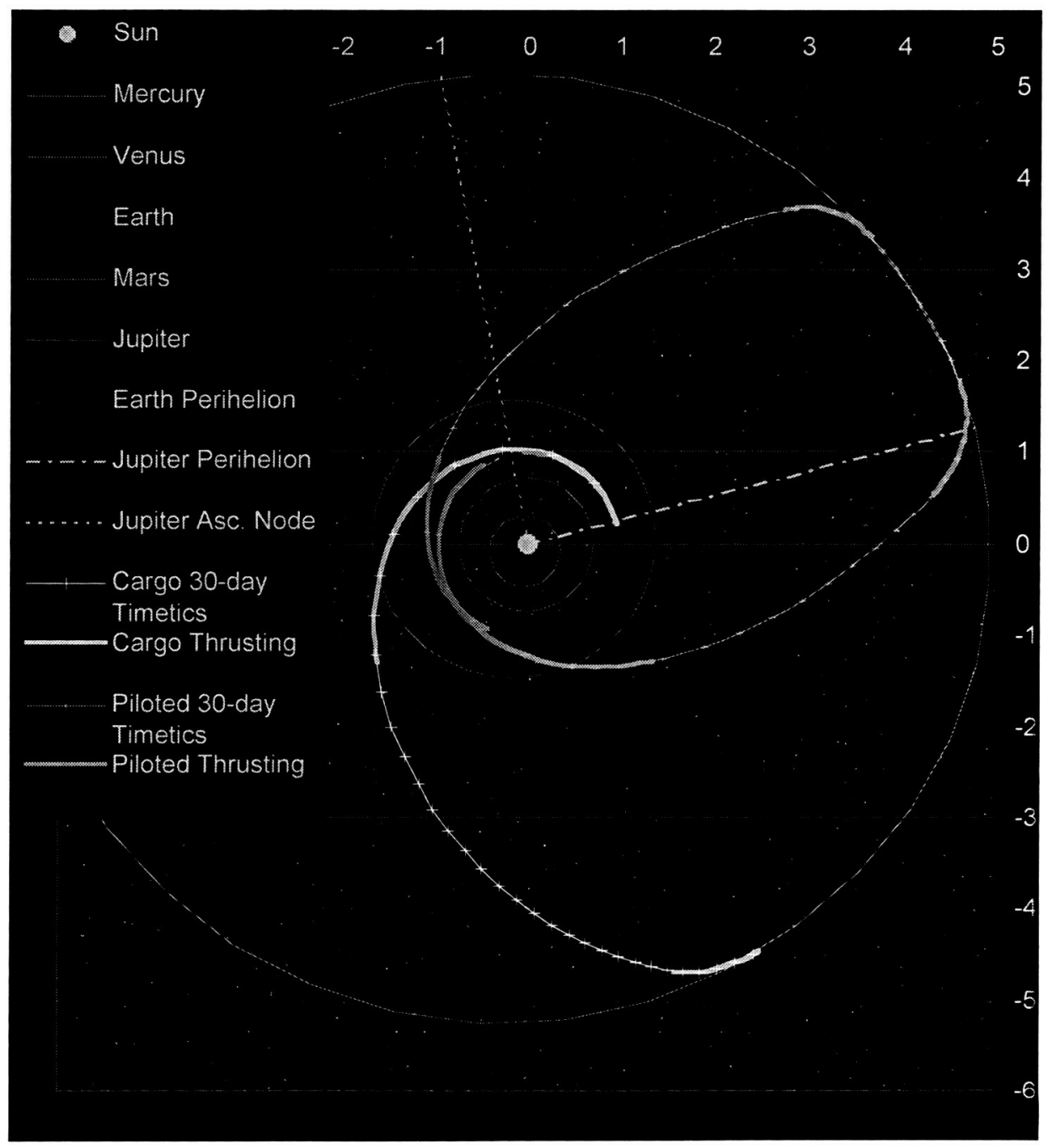

\section{Configuration Design}

The HOPE vehicle configuration is designed for launch on a Delta IV type expendable launch vehicle. Multiple launches and in-space assembly will be required due to the overall size and mass of the vehicle. The payload envelope of the launch vehicle is assumed to be approximately 5.0 meters in diameter by 17.0 meters long.

The major components of the MTF baseline vehicle are the six liquid hydrogen (LH2) tanks, the deuterium tank, the reaction control system (RCS) thrusters and propellant tanks, dual two-sided radiators for high temperature, medium temperature, and crew/avionics heat rejection, a SP-100 reactor, four deuterium-tritium tanks, and a water filled tank which provides radiation shielding for the vehicle. A single magnetized target fusion (MTF) engine is located at the aft end of the vehicle. The HOPE vehicle payload consists of a surface habitat module, a transhab module, a lander, and an ISRU. Structural support of the HOPE vehicle components 
is provided by open truss segments. The main propellant tank cluster has additional support at the forward and aft ends. The payload modules dock to a single node located at the forward end of the vehicle.

Figure 11. MTF D-D 30-Day Stay Vehicle

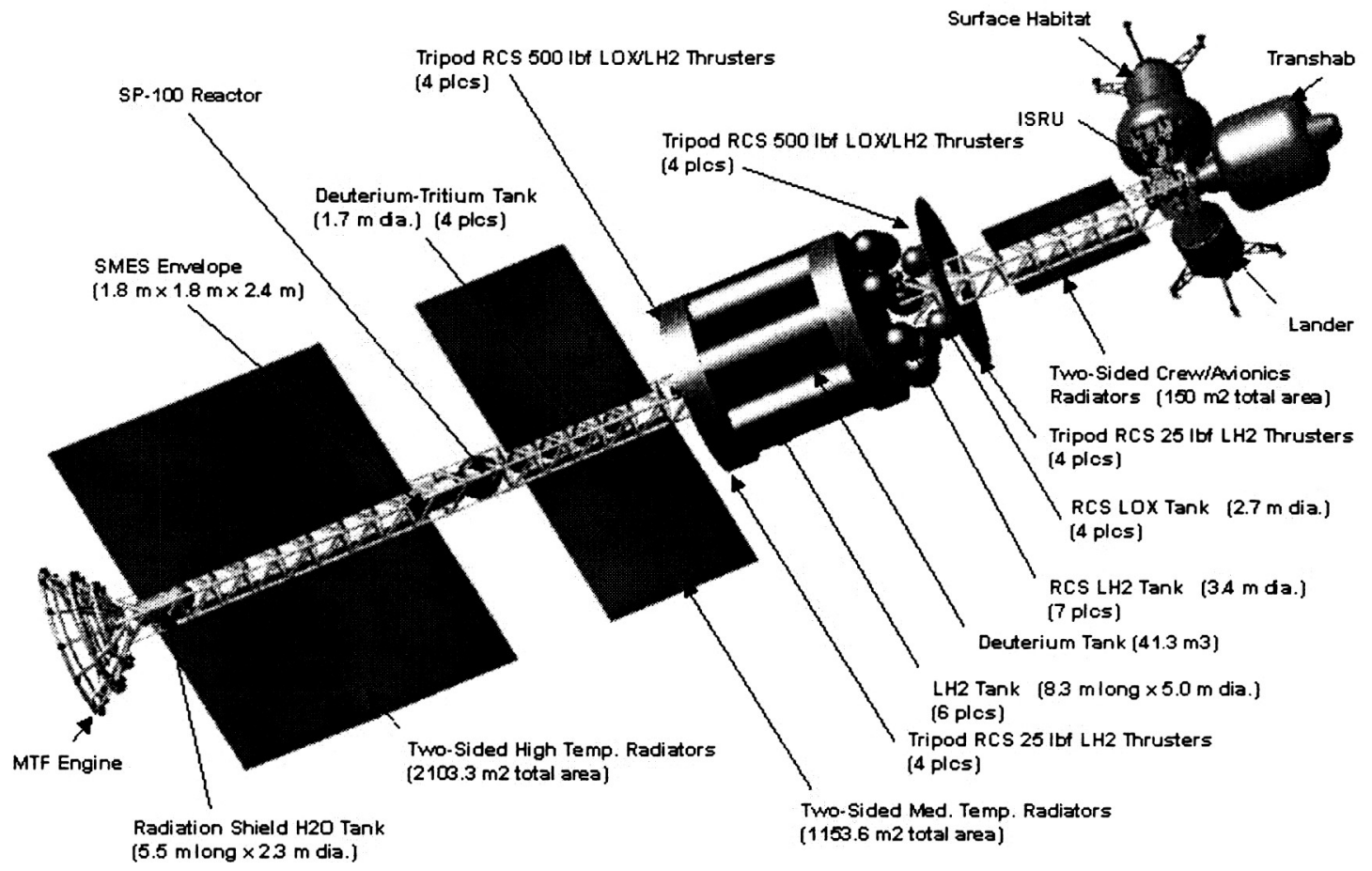

The fully assembled vehicle is approximately the size of a standard football field. The HOPE vehicle overall deployed dimensions are approximately 45 meters in width by 119 meters long. 
Figure 12 MTF D-D 30-Day Stay Vehicle Overall Dimensions

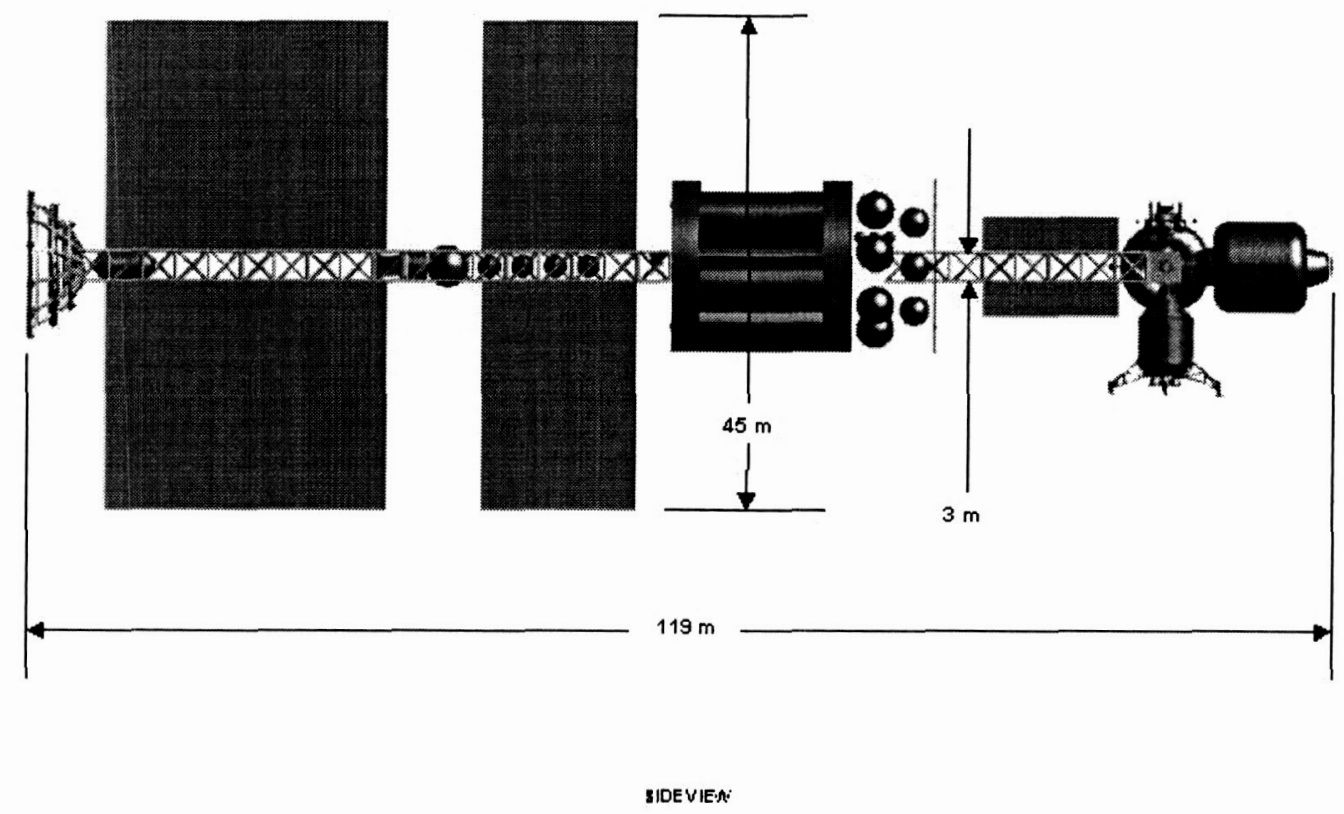

The HOPE vehicle propulsion system components are located in as close proximity as possible to the MTF to minimize the weight and routing complexity of the required propellant lines, coolant lines, and power system cables. 
Figure 13. MTF D-D HOPE Vehicle Aft End Detail

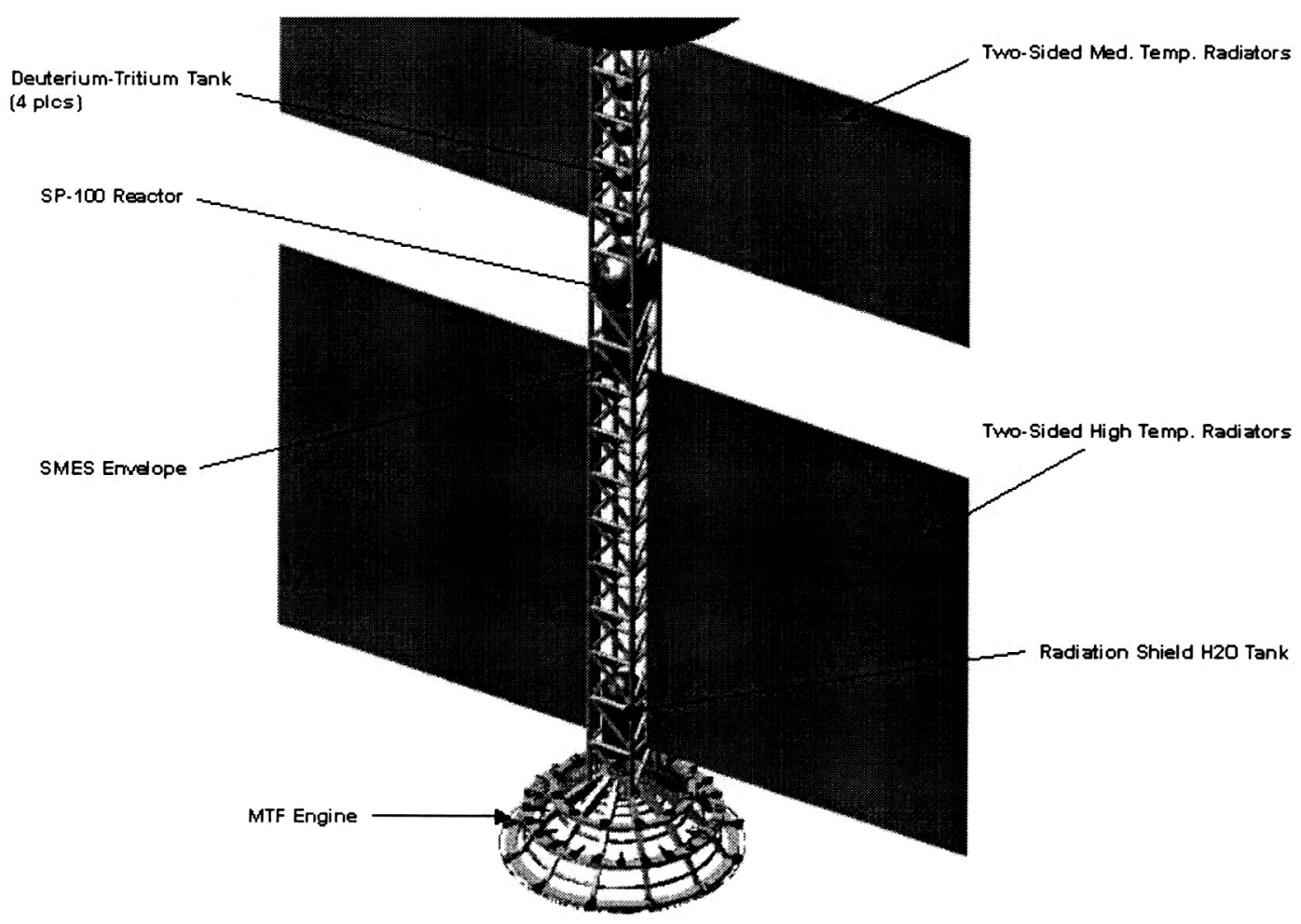

The RCS is located in the proximity of the center of gravity of the vehicle. The RCS thrusters are located at a minimum distance from the RCS propellant tanks to minimize the weight and complexity of the associated plumbing required. The crew/avionics radiators are located adjacent to the payload area to minimize the length of the required coolant lines. 
Figure 14. HOPE Vehicle Forward End Detail

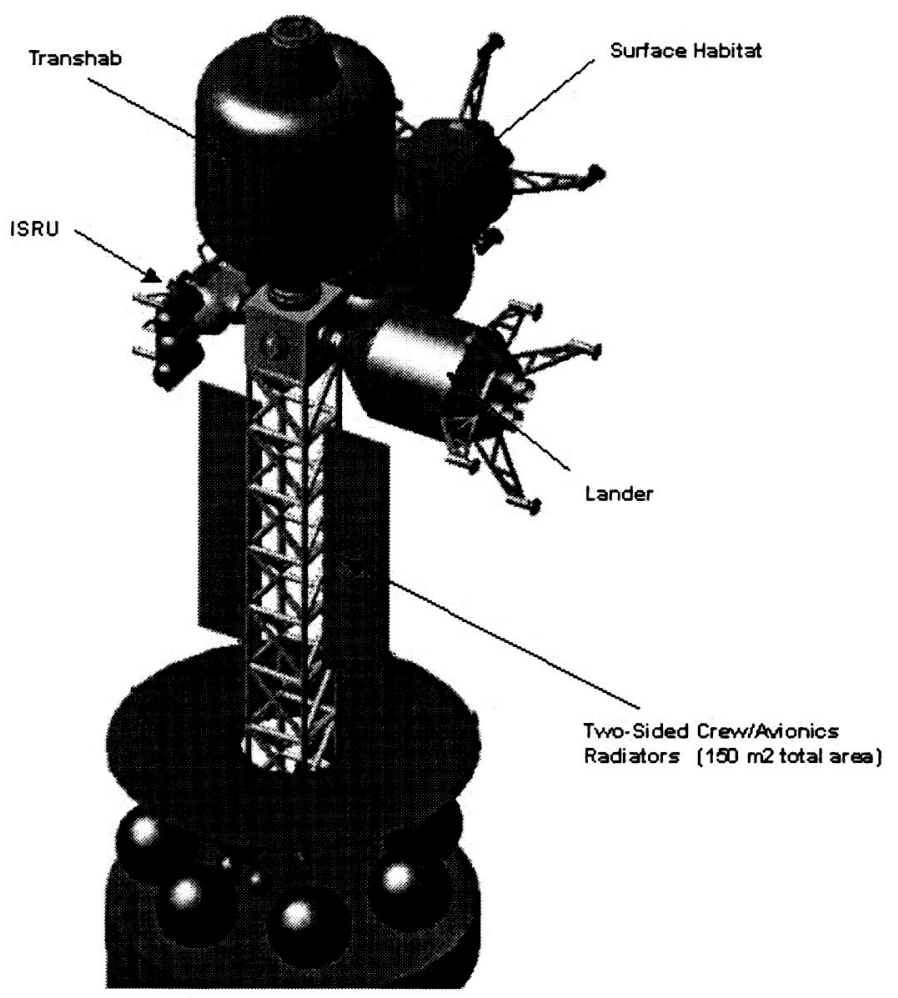


Figure 15. HOPE MTF D-D 180 Day Mission Vehicle Configuration

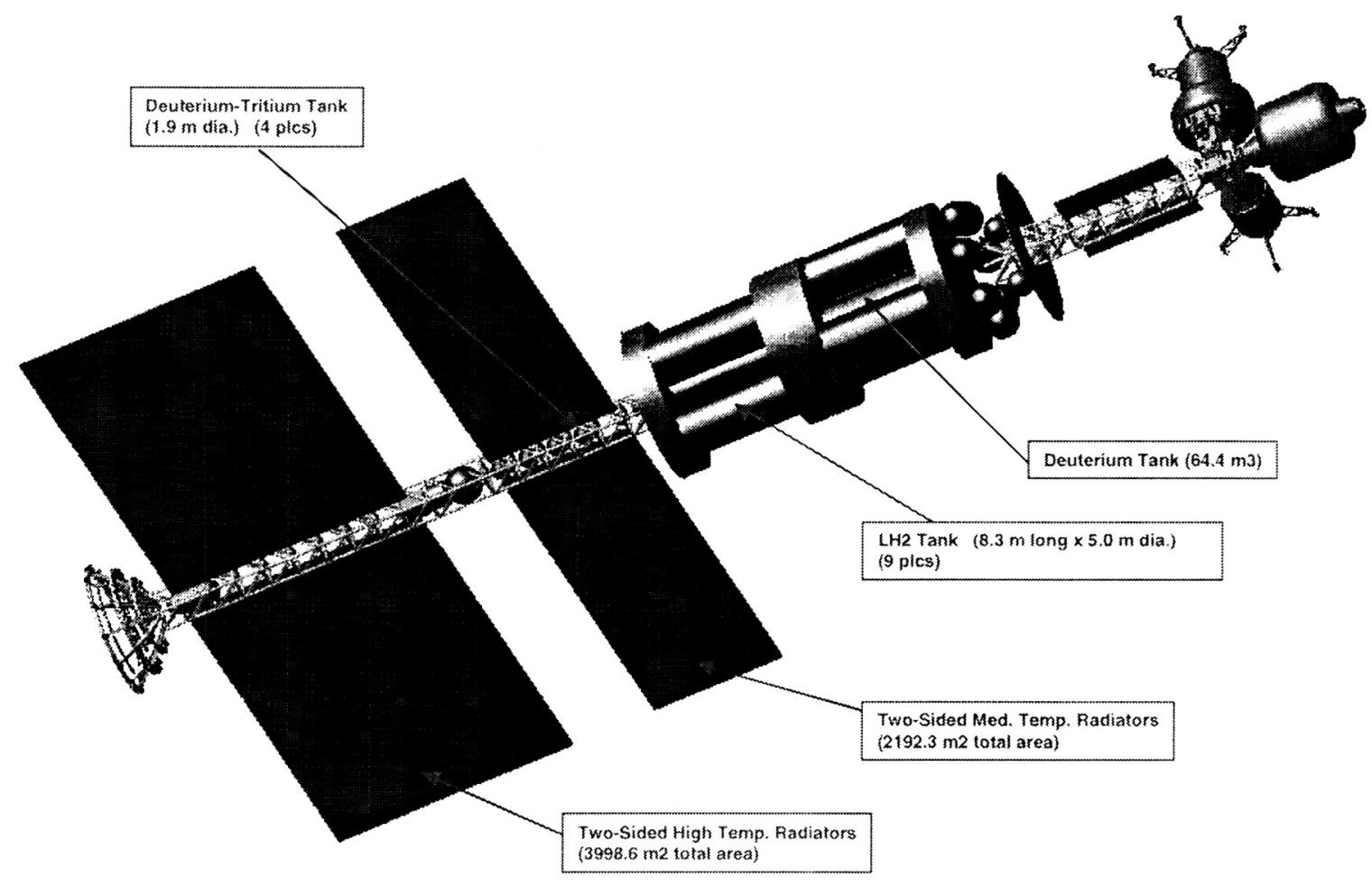


Figure 16. HOPE MTF D-He3 180-Day Mission.

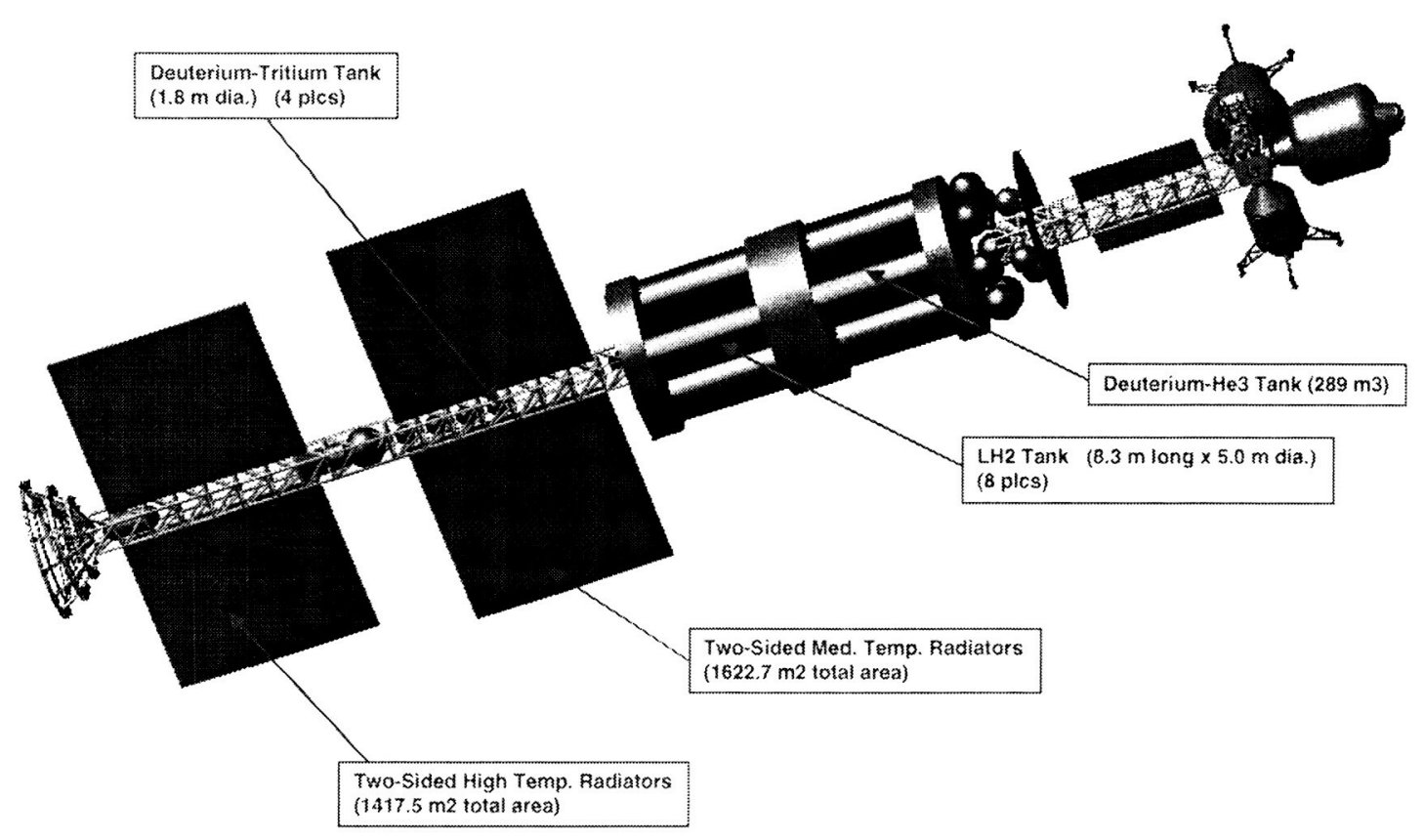

Mass Properties

Table XX Shows the subsystem mass estimates for each of the concepts analyzed for this study.

\begin{tabular}{|l|c|c|c|c|c|c|c|}
\hline & $\begin{array}{c}\text { MTF D-D } \\
\text { 30-day } \\
\text { Stay }\end{array}$ & $\begin{array}{c}\text { MTF D-D } \\
\mathbf{1 8 0 - D a y} \\
\text { Stay }\end{array}$ & $\begin{array}{c}\text { MTF D- } \\
\text { He3 180- } \\
\text { Day Stay }\end{array}$ & $\begin{array}{c}\text { LMR-MPD } \\
\text { Piloted }\end{array}$ & $\begin{array}{c}\text { LMR-MPD } \\
\text { Cargo }\end{array}$ & $\begin{array}{c}\text { MHD-MPD } \\
\text { Piloted }\end{array}$ & $\begin{array}{c}\text { MHD-MPD } \\
\text { Cargo }\end{array}$ \\
\hline Payload & 163,933 & 163,933 & 163,933 & 43,933 & 120,000 & 57,846 & 120,000 \\
\hline Structural & 26,610 & 34,785 & 32,060 & 94,735 & 29,335 & 78,385 & 29,335 \\
\hline Reaction Control System (RCS) & 12,946 & 12,989 & 12,976 & 12,903 & 12,903 & 12,903 & 12,903 \\
\hline Thermal & 51,391 & 76,864 & 51,306 & 50,133 & 20,790 & 42,909 & 20,790 \\
\hline Power & 17,370 & 17,370 & 17,370 & 72,149 & 37,157 & 89,371 & 37,157 \\
\hline Main Propulsion & 116,021 & 121,333 & 118,400 & 58,596 & 17,794 & 13,134 & 17,794 \\
\hline Total Dry Mass & $\mathbf{5 0 4 , 7 5 3}$ & $\mathbf{5 5 5 , 4 5 7}$ & $\mathbf{5 1 4 , 8 5 9}$ & $\mathbf{4 3 2 , 1 8 5}$ & $\mathbf{3 0 9 , 3 7 3}$ & $\mathbf{3 8 2 , 9 1 4}$ & $\mathbf{3 0 9 , 3 7 3}$ \\
\hline IML1 & $\mathbf{6 4 4 , 8 1 6}$ & $\mathbf{7 5 5 , 8 0 5}$ & $\mathbf{6 9 1 , 5 3 5}$ & $\mathbf{1 , 0 4 4 , 5 5 9}$ & $\mathbf{4 3 7 , 2 6 9}$ & $\mathbf{8 7 8 , 2 8 9}$ & $\mathbf{4 3 7 , 2 6 9}$ \\
\hline
\end{tabular}

